\title{
Word length and rarity in recognition memory
}

ARTHUR I. SCHULMAN UNIVERSITY OF VIRGINIA

Operating characteristics were used to assess the importance of word-frequency and word-length in recognition memory. It was much easier for Ss to distinguish between old and new rare words than between old and new common words. Rare (but not common) words were easier to recognize when they were polysyllabic than when they were not.

Recognition memory may be analyzed with the tools of signal detection theory, as Egan (1958) was the first to show. Briefly, the method is to present $\mathrm{S}$ with a sequence of items, in our case English words. When the words later reappear, dispersed in a test sequence among other words not previously presented, $S$ must characterize each word in turn as either "old" or "new." If S's responses are confidence ratings, rather than simply binary decisions, individual operating characteristics (OCs) may be obtained. (See Green \& Swets, 1966, for details and further discussion.) Such an OC represents the proportion of old words judged to be old, i.e., $\mathrm{P}(\mathrm{O} \mid \mathrm{o})$, as a function of the proportion of new words judged to be old, i.e., $\mathrm{P}(\mathrm{O} \mid \mathrm{n})$, and reflects the difficulty in discriminating new words from old.

The experiment reported here used OCs to assess the importance of two variables-word length and rarity-in recognition memory. It was expected that words occurring infrequently in English would be easier to recognize than common words (see Shepard, 1967, and Gorman, 1961, for supporting data), and that polysyllabic words would be easier to recognize than monosyllables. The bases for these expectations will be amplified in a later section, but for the present we may note that (a) the longer an utterance, the less likely it is to resemble another utterance of equal length, and (b) rare words not only may be more specific, concrete, and freer from familiar associations than common words, but also may differ from them structurally.

Method

Unlike most previous studies of word recognition, our words were presented not to the eye but to the ear. ${ }^{1}$ The $E$ read to the Ss a sequence of 100 words at a rate of about 1 word $/ 2 \mathrm{sec}$. After a brief interlude in which Ss were instructed in the use of a 6 -point rating scale (" 1 " = highest confidence the word is old, "6" = highest confidence the word is new, etc.), $\mathrm{E}$ read, at a rate of about 1 word $/ 4 \mathrm{sec}$, a sequence of 200 words that included the original 100. Each $S$ recorded his response on a numbered sheet provided by $\mathrm{E}$. Old and new words alike included 50 words of one syllable and 50 words of three or four syllables. Of each such set of 50 , half were common English words (Thorndike-Lorge, 1944, frequency of
A or AA) and half were comparatively rare (ThorndikeLorge frequency of 1). Each test word thus fell into one of eight disjoint categories, each with 25 representatives: old/new and common/rare and monosyllabic/polysyllabic (OCM, OCP, ORM, ORP, NCM, NCP, NRM, NRP). Subject to these category constraints, words were assigned at random to each set of 25 . The order of the original presentation sequence was random. The test sequence was random but constrained: The first eight words, and all succeeding blocks of eight, had to contain exactly one word of each category, and no block might begin with four new or four old words. The Ss, tested as a group, were 15 male students in an introductory laboratory course in experimental psychology. They were at the time of testing unaware of the structure of the word lists and of the detailed purposes of the experiment.

\section{Results and Discussion}

Before proceeding to the results of primary interest, we pause to examine overall recognition performance. On a double-probability plot, linear OCs for each $\mathrm{S}$ $(n=200)$ fitted the points obtained for old-new discriminations reasonably well. When all responses were pooled and a single "average"' OC was fitted by eye to the points that resulted $(n=3000)$, this linear $O C$ appeared to be representative: its slope $m$ of .75 was the median slope (11 Ss showed slopes between .73 and .84 , four between .40 and .49), and its $d_{S}$ was 1.50 (mean individual $d_{S}$ was 1.60 , median 1.42 ). ${ }^{2}$ What is more, the fit was excellent: the five points were $(.063, .567)$; $(.139, .686) ;(.210, .767)$; (.324, $.823)$; and $(.557, .919)$. Since the average OC for all the words appeared to reflect, and not distort, individual performance, responses were freely pooled in all other analyses described below. The data from only nine of the 15 Ss were available for these detailed analyses.

The effects of the main variables on recognition performance are indicated by the OCs of Fig. 1. It is clear that rare words are much easier than common words to identify as old or new. What is also apparent is that having three or four syllables makes recognition easier for rare words only: compare the wide disparity between the two upper curves and the negligible difference between the two lower curves. (Data from individual Ss show eight of nine to have performed better with RP than with RM; eight of nine better with RM than with $\mathrm{C}$; and five of nine better with $\mathrm{CM}$ than with $\mathrm{CP}$ ). The similar slopes of the linear OCs of Fig. 1 argue that old-new decisions for unequally difficult sets of homogeneous words are made in the same fashion.

The marked superiority in recognition memory for 


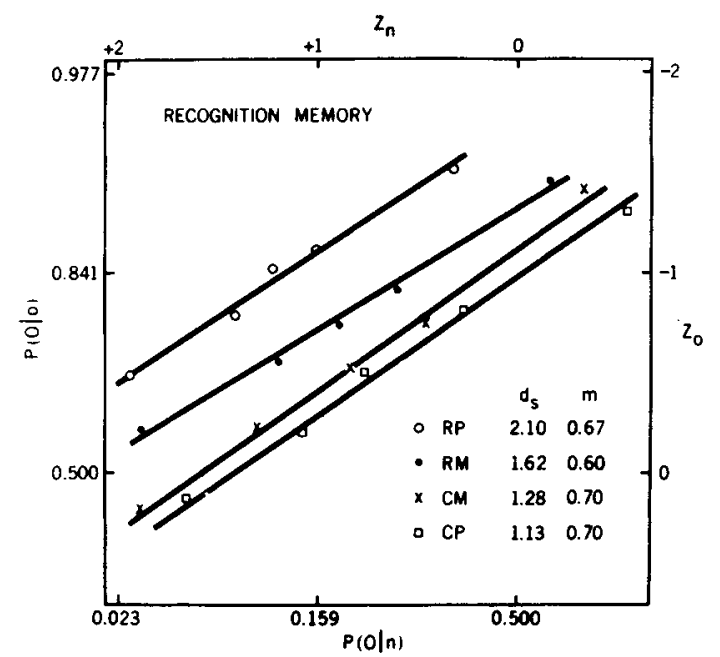

Fig. 1. Operating characteristics on double-probability paper. $(R=$ rare, $C=$ common, $M=$ monosyllabic, $P=$ polysyllabic.) Each $\mathrm{OC}$, fitted by eye, is based on the ratings assigned by nine Ss to 25 old and 25 new words.

rare words is notable when we consider that our "rare" words were by no means unfamiliar ones. Using somewhat more infrequent words and a forcedchoice procedure, Shepard (1967) found comparable differences in the recognizability of rare and common words. He measured performance in terms of the percentage of correct decisions, $p(C)$. The values of $p(C)$ he reports for homogeneous pairings of choice itemsOR VS NR (92.0\%) and OC vs NC (82.1\%)-are each greater by about $3 \%$ than the values our Ss would have been expected to achieve in a forced-choice experiment (see Green \& Moses, 1966). For heterogeneous pairings-OR vS NC and OC vs NR-Shepard reports easier recognition when the rare word is old. The present data corroborate this difference only for strict response criteria; the two OCs, differing markedly in slope $(m=.50$ and .90$)$, intersect near the negative diagonal, so that for lax criteria (associated with the upper portion of the $\mathrm{OC}$ ) recognition is easier when the rare word is new. In terms of $d_{S}$, however, whether the old or new word is rare does not seem to matter.

Why are comparatively rare words easier to recognize than very common ones? For one thing, rare words should tend to be more distinctive semantically, since they should share fewer associations than do common words. For another, it can be shown that rare words differ significantly in structure from common words, for instance in their greater incidence of unusual digrams. The author has been unable to show, however, that Ss base decisions on structural differences, though the failure to do so may owe more to inability to isolate the proper storage units than to actual neglect of structural information by $S$.

Why should a longer word be easier to recognize only when the words in question are rare? Perhaps a plausible answer is that length (and structure generally?) is a secondary property used only after some sort of "semantic search" has been initiated. For rare words this search produces only a few possible matches, so that the additional information provided by length may be helpful. For common words, semantic spaces overlap too much for a structural search to be carried out successfully. Needless to say, all this is highly speculative.

In summary, we have used OCs to show the importance of word-frequency for recognition memory, and the importance of word-length when relatively rare words are involved. But the properties of rare and polysyllabic words that lend them their distinctiveness have yet to be elucidated.

\section{References}

EGAN, J. P. Recognition memory and the operating characteristic. Indiana University: Hearing and Communication Laboratory, Tech. Note AFCRC-TN-58-51, 1958.

GORMAN, A. M. Recognition memory for nouns as a function of abstractness and frequency. J. exp. Psychol., 1961, 61, 23-29.

GREEN, D. M., \& MOSES, F. L. On the equivalence of two recognition measures of short-term memory. Psychol. Bull. 1966, 66, 228-234.

GREEN, D. M., \& SWETS, J. A. Signal detection theory and psychophysics. New York: John Wiley \& Sons, Inc., 1966. Pp. 337-345.

SHEPARD, R. N. Recognition memory for words, sentences, and pictures. J. verbal Learn verbal Behav., 1967, 6, 156-163.

THORNDIKE, E. L., \& LORGE, I. The teacher's word book of 30,000 words. New York: Columbia University Press, 1944.

\section{Notes}

1. This was done for convenience's sake and not for theoretical reasons. The OCs that resulted were similar in form to OCs obtained after visual presentation.

2. The index of discriminability $d_{s}$ is defined as the difference between the $z$-score co-ordinates at the point on the $O C$ that lies on the negative diagonal. 